\title{
On the surface of glasses
}

\author{
Jacob D. Stevenson and Peter G. Wolynes ${ }^{\text {a) }}$ \\ Department of Physics and Department of Chemistry and Biochemistry, Center for Theoretical Biological \\ Physics, University of California, San Diego, La Jolla, California 92093, USA
}

(Received 11 September 2008; accepted 14 November 2008; published online 19 December 2008)

\begin{abstract}
Dynamics near the surface of glasses is generally much faster than in the bulk. Neglecting static perturbations of structure at the surface, we use random first order transition (RFOT) theory to show the free energy barrier for activated motion near a free surface should be half that of the bulk at the same temperature. The increased mobility allows the surface layers to descend much further on the energy landscape than the bulk ordinarily does. The simplified RFOT calculation, however, predicts a limiting value for the configurational entropy a vapor deposited glass may reach as a function of deposition rate. We sketch how mode coupling effects extend the excess free surface mobility into the bulk so that the glass transition temperature is measurably perturbed at depths greater than the naive length scale of dynamic cooperativity. (C) 2008 American Institute of Physics.
\end{abstract}

[DOI: $10.1063 / 1.3041651]$

Paying attention to the properties of interfaces has, since the time of van der Waals, helped to clarify our understanding of bulk phase transitions. ${ }^{1}$ Studying the mobility at the surface of glasses and supercooled liquids has the potential to be equally enlightening about the glass transition. ${ }^{2,3}$ Theories of the glass transition predict a growing length scale of correlated dynamics as the glass transition is approached. ${ }^{4-7}$ This growth, according to the most successful theories, is quite modest. It has only in recent years been widely acknowledged that there is such a slowly growing length scale in bulk glasses. ${ }^{8,9}$ The dynamics of a glass or supercooled liquid should be perturbed within a few correlation lengths of its surface. Many experiments do show significant perturbations of the glassy dynamics at free surfaces or in confined spaces. ${ }^{10}$ The picture that emerges from these experiments is, at present, still somewhat confused. In general, mobility seems to be increased at a free surface, although sometimes a diminished mobility has been observed. At interfaces with solids both increases and decreases in mobility have been reported. ${ }^{11,12}$ It seems likely that this complex set of behaviors reflects the fact that the dynamics of the liquid can be strongly influenced by the liquid's static structure, which also will be perturbed by the interface-in the extremes, partial crystallization can occur at a free interface while a drying layer may sometimes insulate a confined fluid from its solid surroundings. While noting these complications, we feel it is nevertheless worthwhile to present a simplified view of glass and supercooled liquid surfaces in the context of the random first order transition (RFOT) theory of the glass transition.

The RFOT theory is a constructive approach to structural glass dynamics that has explained quantitatively numerous bulk glass phenomena. ${ }^{4}$ It was recognized very early on that growing length scales should be associated with an ideal glass transition as envisaged in RFOT theory. ${ }^{13,14}$ Indeed some of the early experiments on confined supercooled liq-

${ }^{a)}$ Electronic mail: pwolynes@ucsd.edu. uids carried out by Zhang et al. ${ }^{15}$ were motivated by a desire to test these expectations. It turns out that present day RFOT theory for molecular liquids can make somewhat more definite predictions than was done in those early days, at least for an idealized interface that can be taken to have no change in static structure from the bulk. We show for this idealization that the maximum mobility at a completely free interface is related in a very simple way to the bulk mobility. In terms of relaxation times, the RFOT result for the surface relaxation time is simply $\tau_{\text {surf }}=\sqrt{\tau_{0} \tau_{\text {bulk }}}$. We also show that RFOT theory suggests that as measured by an effective local glass transition temperature $T_{g}^{\text {local }}$ the influence of the interface can appear to be rather far reaching into the bulk, consistent with some experiments.

At the smallest length scales the dynamic arrest of glasses results from the cage effect in which a particle's motion is constrained by the presence of its neighbors. In mean field theory this leads to a friction crisis at the mode coupling theory (MCT) critical temperature and the emergence of an ensemble of aperiodic crystal structures having an extensive configurational entropy $s_{c}$ per particle. Below the dynamical transition at $T_{c}$ any large scale motion that takes place is necessarily collaborative. Near a free surface this picture is modified. Since surface particles feel a weaker structural cage, essentially only on the inner side, they would go through a dynamic arrest at a lower temperature and remain more mobile below the bulk glass transition temperature. The collaborative dynamics propagates the enhanced mobility at the surface some distance into the bulk. This depth would be determined by the length scale of cooperativity.

In the mean field limit (made precise in Kac models by Franz and Montanari ${ }^{16}$ ) two cooperative length scales can be defined for RFOT theory. ${ }^{14}$ One scale, $\xi_{\mathrm{MCT}}$, is directly related to the dynamical transition at $T_{c}$, which resembles a spinodal, ${ }^{7}$ and the other is related to the size of regions that rearrange by activated motions and scales with the configurational entropy $s_{c}{ }^{17}$ The latter scale diverges at the Kauzmann temperature $T_{K}$, the temperature where the bulk con- 
figurational entropy becomes nonextensive, as $\xi_{\mathrm{RFOT}} \sim a s_{c}^{\psi}$. Within the sharp, thin wall approximation the RFOT theory yields a numerical coefficient $a$ that appears consistent with experimental values for molecular glasses. ${ }^{17}$ The scaling exponent used to fit inferred lengths from $\chi_{4}$ is also not inconsistent with the RFOT result $\psi=2 / 3$, although a larger value provides a better fit. ${ }^{8,9}$ For molecular liquids governed by short range interactions the dynamical (mode coupling) length would not actually diverge at $T_{c}$ because of a dynamical cutoff from the activated events and will not be too different from $\xi_{\text {RFOT }}$.

We first review the theory of bulk activated dynamics. ${ }^{4}$ Below the MCT transition, dynamics takes place on a rugged free energy landscape. ${ }^{14,18,19}$ Particle motion occurs locally through transitional hops between metastable structural states that resemble, in many ways, nucleation processes between different aperiodic crystal structures. ${ }^{14,17}$ The magnitude of the free energy barrier can be found by a competition between the entropic cost of remaining confined to one minimum free energy structure and a mismatch free energy penalty, $\sigma$, for having two mean field solutions adjacent to each other but in distinct structural states. The free energy profile governing nucleation of a spherical cooperative region of radius $r$ is

$$
F_{\text {bulk }}(r)=4 \pi r^{2}\left(r_{0} / r\right)^{1 / 2} \sigma_{0}-\frac{4}{3} \pi r^{3} n_{0} T s_{c} .
$$

The surface penalty $\sigma(r)=\left(r_{0} / r\right)^{1 / 2} \sigma_{0}$ scales with $r$ due to renormalization effects of wetting by the diverse set of structures. ${ }^{17,20}$ There is some discussion in the community about the value of the surface scaling exponent. ${ }^{9,21}$ In the absence of wetting or when it is incomplete, a weaker variation of $\sigma(r)$ is expected. In any event, at short length scales, $\sigma_{0}$ can be obtained from a crude density functional calculation giving $\sigma_{0}=\frac{3}{4} k_{B} \operatorname{Tr}_{0}^{-2} \ln \left(1 /\left(d_{L}^{2} \pi e\right)\right)$ where $d_{L} \approx 0.1$ particle spacing is the Lindemann length, and $n_{0}=r_{0}^{-3}$ is the density of the liquid. The configurational entropy, $s_{c}$, measures the number of available structural states. The resulting free energy barrier for reconfiguration events in bulk, assuming wetting, is

$$
F_{\text {bulk }}^{\ddagger}=\frac{3 \pi \sigma_{0}^{2} r_{0}^{4}}{T s_{c}(T)} .
$$

The relaxation time $\tau_{\alpha}=\tau_{0} e^{F^{\dot{亠}} / k_{B} T}$ diverges at the Kauzmann temperature $\left(s_{c}\left(T_{K}\right) \rightarrow 0\right)$ and thus follows the VogelFulcher behavior in the deeply supercooled region $\tau_{\alpha}$ $\sim e^{B /\left(T-T_{K}\right)}$. The length scale of an activated event follows from balancing the terms in the free energy profile

$$
r^{*}=r_{0}\left(\frac{3 \sigma_{0} r_{0}^{2}}{T s_{c}(T)}\right)^{2 / 3}
$$

This length scale increases with decreasing temperature, reaching a universal value of about five interparticle spacings at the glass transition temperature corresponding to a one hour relaxation time.

The RFOT analysis of bulk activated dynamics can be easily modified (see Fig. 1) to treat motions near a free surface. Near a completely free surface with no structural modifications, the transition state would rearrange a region of

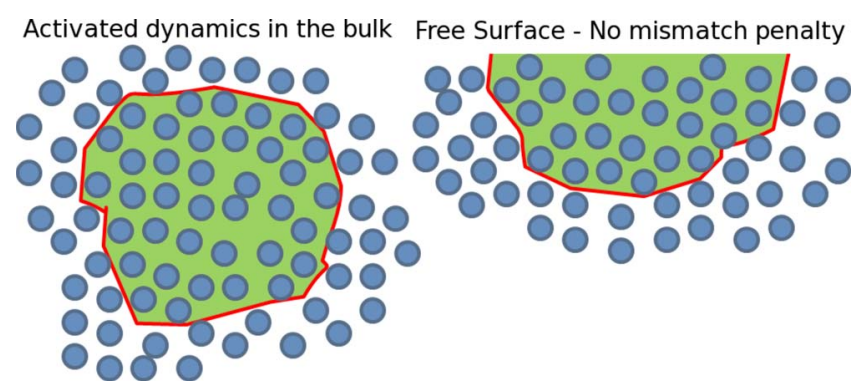

FIG. 1. (Color online) In the bulk, at low temperatures, activated motion occurs within roughly spherical regions. Near a free surface a rearranging hemispherical region feels no mismatch penalty on interface along the free surface leading to much faster dynamics than the bulk.

hemispherical shape in order to minimize the surface area subject to the mismatch penalty (for shapes other than hemispheres the wetting effect would need to be modified to correctly deal with the curvature, but the hemisphere, or something close to it, would still be the most favored shape). The flat face of the hemisphere lies along the free surface and is assumed not to contribute to the mismatch penalty. The free energy profile for the hemispherical rearranging region becomes

$$
F_{\text {surf }}(r)=2 \pi r^{2}\left(r_{0} / r\right)^{1 / 2} \sigma_{0}-\frac{2}{3} \pi r^{3} n_{0} T s_{c} .
$$

Since both terms in the expression are halved from the equation for bulk dynamics the size corresponding to the transition state for activated rearrangement remains unchanged from that for the bulk, but the resulting free energy barrier is reduced by a factor of 2 ,

$$
F_{\text {surf }}^{*}=\frac{1}{2} \frac{3 \pi \sigma_{0}^{2} r_{0}^{4}}{T s_{c}(T)} .
$$

This seemingly innocuous change leads to dramatically faster relaxation at free surfaces than occurs in the bulk. Molecular motion near the surface of a glass would still be detectable even if the bulk were essentially frozen. Very near the surface, the relaxation time is related very simply to the bulk value,

$$
\tau_{\text {surf }}=\sqrt{\tau_{0} \tau_{\text {bulk }}} .
$$

This result is independent of the wetting.

According to RFOT, on laboratory time scales, the ideal surface layer will be able to "hike" (using the colorful expression of Kearns et $a .^{22}$ ) much further down the free energy landscape than the bulk, reaching lower energy, more stable configurations than the bulk is likely to find. The surface motion will freeze out, i.e., go through its own glass transition $\left(T_{g}^{\text {surf }}\right)$ only when $\tau_{\text {surf }}$ becomes larger than the laboratory time scale. For the same laboratory time scale, according to Eq. (5), this will happen when the configurational entropy is half the characteristic configurational entropy of the bulk glass transition. The bulk glass transition entropy is very nearly material independent, ${ }^{23,24}$ and likewise the free surface glass transition will occur at a characteristic bulk entropy value. For a one hour time scale this characteristic entropy is

$$
s_{c}\left(T_{g}^{\text {surf }}\right)=\frac{1}{2} s_{c}\left(T_{g}^{\text {bulk }}\right) \approx 0.41 k_{B} .
$$


Swallen et $a l^{2,22}$ recently showed how to construct a macroscopic glass sample via vapor deposition reaching much lower in the energy landscape than is possible by the usual bulk cooling of a liquid. They argued that this ultrastable glass arises from the free surface's excess mobility. For slow deposition on a cold substrate the growing glass, owing to the higher mobility, can more thoroughly rearrange near the free surface to form exceedingly stable structures. They assigned a fictive temperature to their sample, $T_{f}$, defined as the temperature where a glass of similar stability, but created by bulk cooling, would fall out of equilibrium. $T_{f}$ can be used to measure the sample's position on the free energy landscape relative to an ordinary glass through the parameter

$$
\theta_{K}=\frac{T_{g}-T_{f}}{T_{g}-T_{K}} .
$$

This parameter increases with the stability of the glass, reaching one for an ideal glass. In principle if there were, in fact, no entropy crisis, $\theta_{K}$ could exceed one. $\theta_{K}$ can be parametrized by the configurational entropy, an equivalent measure of structural stability. Using the linear extrapolation vanishing at $T_{K}$ valid for low temperature $\left(T<T_{g}\right)$ the RFOT free surface mobility would yield for a rate of deposition equal to one correlation length per hour

$$
\theta_{K}=1-\frac{s_{c}\left(T_{f}\right)}{s_{c}\left(T_{g}\right)} \leq \frac{1}{2} .
$$

As the enhanced surface mobility freezes out at $T_{g}^{\text {surf }}$, this temperature should be seen as a limiting value for $T_{f}$ leading to the inequality above. The experiments of Swallen et $a l^{2,22}$ yielded $\theta_{K} \approx 0.4$ for the two liquids studied. It would take about $10^{5}$ years to make these highly stable glasses via traditional bulk cooling, while roughly the age of the universe would be required for a glass with $\theta_{K}=1 / 2$. Unless the surface structure is greatly modified leading to a locally smaller $T_{K}$, vapor deposition should not yield glasses with stability much greater than the naive RFOT limiting value.

The linear extrapolation for $s_{c}(T)$ is valid near $T_{K}$, but for some liquids at higher temperatures a better approximation is ${ }^{25} s_{c}=s_{\infty}\left(1-T_{K} / T\right)$. Recognizing this feature does add some material dependence to the limiting stability

$$
\theta_{K} \leq \frac{T_{g}}{T_{g}+T_{K}} .
$$

Fragile liquids still have a limit near $1 / 2$, but strong liquids have the possibility of descending further in terms of temperature (but not in terms of configurational entropy) than fragile liquids. The fragilities for the two glasses so far studied by Swallen et al. ${ }^{2,22}$ are similar and would yield $\theta_{K}$ $\leq 0.57(\mathrm{IMC})$ and $\theta_{K} \leq 0.58(\mathrm{TNB})$ for the nanometer per hour deposition rate.

A key prediction of this analysis is that the fictive temperature is a function of the logarithm of the deposition rate. More precisely the configurational entropy at the fictive temperature is a universal function of the logarithmic deposition rate. The limiting stability given above assumes a deposition rate of one correlation length per hour. More generally, for a deposition rate $k$, the mobile surface layer of depth $\xi$ will be

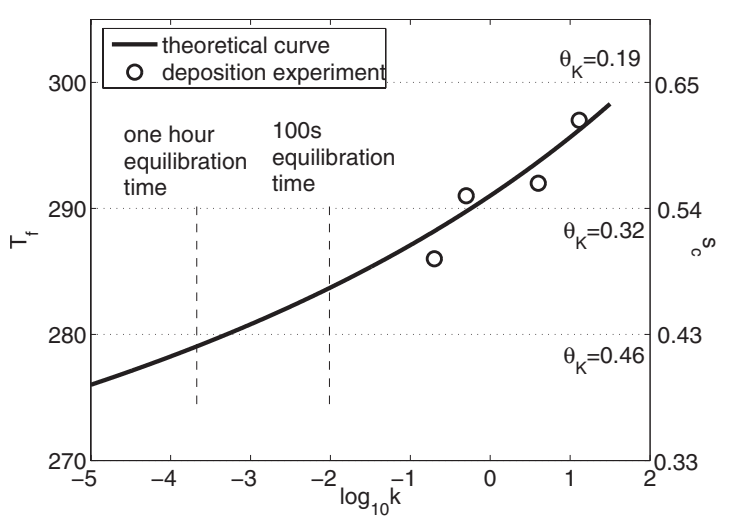

FIG. 2. Fictive temperature vs deposition rate for the glass former IMC. Data for the deposition experiment were taken from Ref. 22. Fictive temperatures are converted into configurational entropy (right axis) and stability, $\theta_{K}$, for comparison.

equilibrated on a time scale $\xi / k$, which is related to the fictive temperature through the relationship $\xi / k$

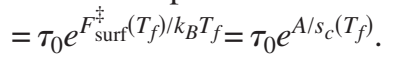

$$
s_{c}\left(T_{f}\right)=\frac{A_{\text {surf }} \log _{10} e}{\log _{10}\left(\xi / k \tau_{0}\right)} .
$$

We compared it to the experimental results of Kearns et $a l^{22}$ using the linear representation for the configurational entropy, $s_{c}\left(T_{f}\right)=\Delta C_{p}\left(T_{g}^{\text {bulk }}\right)\left(T_{f}-T_{K}\right) / T_{K}$. Along with $A_{\text {surf }}$ obtained from Eq. (5), we take $\xi \approx 1 \mathrm{~nm}$ and $\tau_{0}=10^{-12} \mathrm{~s}$. $\Delta C_{P}\left(T_{g}^{\text {bulk }}\right) \approx 2.6 k_{B}$ is the heat capacity jump at the glass transition for indomethacin (IMC) in Boltzmann units per bead. ${ }^{24}$ The theoretical results along with the measured values obtained by Kearns et al. ${ }^{22}$ are plotted in Fig. 2. Our results are consistent with the experimental data for the deposition rates tested. The theory correctly predicts the trend for slower deposition rates. Despite the good agreement we point out that this is an approximate result in which we have assumed equilibrium relaxation, strictly true only for $T_{\text {substrate }}=T_{f}$.

It would seem natural to say that the enhanced mobility at the surface would penetrate into the bulk at least on the length scales of the bulk cooperative motion, as indeed it does. This, however, results in a local relaxation time $\tau_{\text {local }}(z)$, which changes by many orders of magnitude over just a few particle spacings. This mobility gradient would be relaxed via the dynamics of MCT, diffusing the excess mobility deeper into the bulk. In first approximation MCT has been shown to correlate dynamics on the length scale $\xi_{\mathrm{MCT}}{ }^{7}$ the predicted divergence of which at $T_{c}$ will be broken by the emergence of activated events. ${ }^{26,27} \mathrm{~A}$ simple equation describing this smoothing of the mobility field, incorporating activated events in the framework of Bhattacharyya et al., ${ }^{26}$ emerges,

$$
\xi_{\mathrm{MCT}}^{2} \nabla^{2} \tau^{-1}(z)=\tau^{-1}(z)-\tau_{\text {local }}^{-1}(z) .
$$

If we treat the mobility profile from purely activated dynamics, $\tau_{\text {local }}^{-1}(z)$, as a set of boundary conditions at $z=0$ and $z$ $=\infty$ (equivalent to coarse graining on the length scale of the cooperative motion), then the mobility, decaying smoothly from $\tau_{\text {surf }}^{-1} \propto e^{-A_{\text {surf }} / s_{c}}$ on the free surface to $\tau_{\text {bulk }}^{-1} \propto e^{-A_{\text {bulk }} / s_{c}}$ 
$\ll \tau_{\text {surf }}^{-1}$ in the bulk on a length scale $\xi_{\mathrm{MCT}}$, would follow

$$
\tau^{-1}(z) \approx\left(\tau_{\text {surf }}^{-1}-\tau_{\text {bulk }}^{-1}\right) e^{-z / \xi_{\mathrm{MCT}}}+\tau_{\text {bulk }}^{-1} .
$$

The distance the excess mobility penetrates, $z^{*}$, is found by comparing the magnitude of the two terms.

$$
z^{*}=\xi_{\mathrm{MCT}} \frac{A_{\text {bulk }}-A_{\text {surf }}}{s_{c}} .
$$

We have shown that $A_{\text {surf }}=\frac{1}{2} A_{\text {bulk }}$, and that $A_{\text {bulk }} / s_{c}\left(T_{g}\right) \approx 40$ at the glass transition. This gives a length scale $z^{*} \approx 20 \xi_{\mathrm{MCT}}$, which can be much larger than the bare dynamical correlation length.

At a depth $z<z^{*}$ from the surface the particle motion would freeze out when $\tau^{-1}(z) \approx e^{-A_{\text {surf }} / s_{c}\left(T_{g}^{\text {local }}\right)} e^{-z / \xi_{\text {MCT }}}$ $\approx \tau_{\text {bulk }}^{-1}\left(T_{g}\right)$ or when

$$
\frac{A_{\text {surf }}}{s_{c}\left(T_{g}^{\text {local }}\right)}+\frac{z}{\xi_{\mathrm{MCT}}}=\frac{A_{\text {bulk }}}{s_{c}\left(T_{g}\right)} \text {. }
$$

We find that for the thinnest films the local glass transition temperature will be $T_{g}^{\mathrm{min}}=T_{g}^{\text {surf }}$ as expected. $T_{g}^{\text {local }}$ will grow with distance from the free surface and be indistinguishable from the bulk at a depth $z^{*}$, on the order of $20 \mathrm{~nm}$ in IMC.

The surface properties of glasses are important in many technological and biological contexts. Enhanced surface mobility is relevant for adhesion, friction, coatings, and nanoscale fabrication such as etching and lithography. Supercooled water at the surface of proteins acts to enslave many protein motions. $^{28,29}$ Despite the influence of static surface perturbations, we feel that the idealized treatment of the surface mobility of glasses presented here can help in understanding these phenomena. The enhanced mobility at free surfaces will allow phase transformations to occur at the surface that are kinetically impossible in bulk. Deitrification often occurs at free glass surfaces, a fact of some importance in geology $^{30}$ and archeology. ${ }^{31}$ This observation is naturally explained by the RFOT theory.

Support from NSF Grant No. CHE0317017 and NIH Grant No. 5R01GM44557 is gratefully acknowledged.
${ }^{1}$ J. S. Rowlinson and B. Widom, Molecular Theory of Capillarity (Oxford University Press, New York, 1982).

${ }^{2}$ S. F. Swallen, K. L. Kearns, M. K. Mapes, Y. S. Kim, R. J. Mcmahon, M. D. Ediger, T. Wu, L. Yu, and S. Satija, Science 315, 353 (2007).

${ }^{3}$ Z. Fakhraai and J. A. Forrest, Science 319, 600 (2008).

${ }^{4}$ V. Lubchenko and P. G. Wolynes, Annu. Rev. Phys. Chem. 58, 235 (2007).

${ }^{5}$ D. Kivelson, S. A. Kivelson, X. L. Zhao, Z. Nussinov, and G. Tarjus, Physica A 219, 27 (1995).

${ }^{6}$ S. Franz and G. Parisi, J. Phys.: Condens. Matter 12, 6335 (2000)

${ }^{7}$ G. Biroli and J. P. Bouchaud, Europhys. Lett. 67, 21 (2004).

${ }^{8}$ L. Berthier, G. Biroli, J. P. Bouchaud, L. Cipelletti, D. El Masri, D. L'Hote, F. Ladieu, and M. Pierno, Science 310, 1797 (2005).

${ }^{9}$ S. Capaccioli, G. Ruocco, and F. Zamponi, J. Phys. Chem. B 112, 10652 (2008).

${ }^{10}$ M. Alcoutlabi and G. B. Mckenna, J. Phys.: Condens. Matter 17, R461 (2005).

${ }^{11}$ J. A. Forrest, K. Dalnokiveress, J. R. Stevens, and J. R. Dutcher, Phys. Rev. Lett. 77, 2002 (1996).

${ }^{12}$ C. H. Li, T. Koga, J. Jiang, S. Sharma, S. Narayanan, L. B. Lurio, Y. Hu, X. Jiao, S. K. Sinha, S. Billet, D. Sosnowik, H. Kim, J. C. Sokolov, and M. H. Rafailovich, Macromolecules 38, 5144 (2005).

${ }^{13}$ T. R. Kirkpatrick, D. Thirumalai, and P. G. Wolynes, Phys. Rev. A 40, 1045 (1989)

${ }^{14}$ T. R. Kirkpatrick and P. G. Wolynes, Phys. Rev. B 36, 8552 (1987).

${ }^{15}$ J. Zhang, G. Liu, and J. Jonas, J. Phys. Chem. 96, 3478 (1992).

${ }^{16}$ S. Franz and A. Montanari, J. Phys. A: Math. Theor. 40, F251 (2007).

${ }^{17}$ X. Xia and P. G. Wolynes, Proc. Natl. Acad. Sci. U.S.A. 97, 2990 (2000).

${ }^{18}$ M. Mézard and G. Parisi, Phys. Rev. Lett. 82, 747 (1999).

${ }^{19}$ Y. Singh, J. P. Stoessel, and P. G. Wolynes, Phys. Rev. Lett. 54, 1059 (1985).

${ }^{20}$ J. Villain, J. Phys. 46, 1843 (1985).

${ }^{21}$ J. P. Bouchaud and G. Biroli, J. Chem. Phys. 121, 7347 (2004).

${ }^{22}$ K. L. Kearns, S. F. Swallen, M. D. Ediger, T. Wu, Y. Sun, and L. Yu, J. Phys. Chem. B 112, 4934 (2008).

${ }^{23}$ V. Lubchenko and P. G. Wolynes, J. Chem. Phys. 119, 9088 (2003).

${ }^{24}$ J. D. Stevenson and P. G. Wolynes, J. Phys. Chem. B 109, 15093 (2005).

${ }^{25}$ R. Richert and C. A. Angell, J. Chem. Phys. 108, 9016 (1998).

${ }^{26}$ S. M. Bhattacharyya, B. Bagchi, and P. G. Wolynes, Phys. Rev. E 72, 031509 (2005).

${ }^{27}$ S. M. Bhattacharyya, B. Bagchi, and P. G. Wolynes, Proc. Natl. Acad. Sci. U.S.A. 105, 16077 (2008)

${ }^{28}$ V. Lubchenko, P. G. Wolynes, and H. Frauenfelder, J. Phys. Chem. B 109, 7488 (2005).

${ }^{29}$ H. Frauenfelder, S. G. Sligar, and P. G. Wolynes, Science 254, 1598 (1991).

${ }^{30}$ R. R. Marshall, Geol. Soc. Am. Bull. 72, 1493 (1961).

${ }^{31}$ W. T. Richards, J. Chem. Phys. 4, 449 (1936). 